\title{
Safety and efficacy of nivolumab plus recombinant human endostatin in previously treated advanced non-small-cell lung cancer
}

\author{
Weize Lv ${ }^{1,2 \#}$, Xiaofeng Pei ${ }^{2,3 \#}$, Wenhua Zhao ${ }^{4 \#}$, Yunyan Cong ${ }^{2,3}$, Yajun Wei ${ }^{1,2}$, Ting Li ${ }^{2,3}$, Hongyu Zhang ${ }^{2,5}$, \\ Zhong Lin ${ }^{2,3}$, Yuichi Saito ${ }^{6}$, Jae Jun Kim ${ }^{7}$, Zibin Liang ${ }^{2,3}$, Beilong Zhong ${ }^{2,8}$, Zhihui Wang ${ }^{2,3}$ \\ ${ }^{1}$ Department of Interventional Medicine, The Fifth Affiliated Hospital Sun Yat-sen University, Zhuhai, China; ${ }^{2}$ Guangdong Provincial Key \\ Laboratory of Biomedical Imaging, The Fifth Affiliated Hospital, Sun Yat-sen University, Zhuhai, China; ${ }^{3}$ Department of Thoracic Oncology, \\ The Fifth Affiliated Hospital of Sun Yat-sen University, Zhuhai, China; ${ }^{4}$ Medical Oncology of Respiratory, Affiliated Tumor Hospital of Guangxi \\ Medical University, Nanning, China; ${ }^{5}$ Department of Abdominal Oncology, The Fifth Affiliated Hospital of Sun Yat-sen University, Zhuhai, China; \\ ${ }^{6}$ Department of Surgery, Teikyo University School of Medicine, Tokyo, Japan; ${ }^{7}$ Department of Thoracic and Cardiovascular Surgery, Uijeongbu St. \\ Mary's Hospital, The Catholic University of Korea, College of Medicine, Seoul, Republic of Korea; ${ }^{8}$ Department of Cardiothoracic Surgery, The \\ Fifth Affiliated Hospital of Sun Yat-sen University, Zhuhai, China \\ Contributions: (I) Conception and design: W Lv, X Pei, W Zhao, Z Liang, B Zhong; (II) Administrative support: H Zhang, Z Lin; (III) Provision \\ of study materials or patients: W Lv, X Pei, W Zhao, Y Cong, Y Wei, T Li, Z Lin, Z Liang, B Zhong, Z Wang; (IV) Collection and assembly of \\ data: All authors; (V) Data analysis and interpretation: W Lv, Z Wang; (VI) Manuscript writing: All authors; (VII) Final approval of manuscript: All \\ authors. \\ "These authors contributed equally to this work. \\ Correspondence to: Zibin Liang. Department of Thoracic Oncology, The Fifth Affiliated Hospital of Sun Yat-sen University, Zhuhai 519000, China. \\ Email: liangzb@mail.sysu.edu.cn; Beilong Zhong. Department of Cardiothoracic Surgery, The Fifth Affiliated Hospital of Sun Yat-sen University, \\ Zhuhai 519000, China. Email: beilongzhuhai@hotmail.com; Zhihui Wang. Department of Thoracic Oncology, The Fifth Affiliated Hospital of Sun \\ Yat-sen University, Zhuhai 519000, China. Email: wangzhh33@mail.sysu.edu.cn.
}

Background: Evidence of the efficacy of immune checkpoint inhibitors (ICIs) plus antiangiogenic drugs in previously treated patients with advanced non-small-cell lung cancer (NSCLC) is still insufficient, so we investigated the safety and efficacy of nivolumab plus recombinant human (rh)-endostatin in such patients.

Methods: Patients without epithelial growth factor receptor (EGFR) or anaplastic lymphoma kinase (ALK) targetable mutations in advanced NSCLC who did not respond to previous treatment were enrolled. Eligible patients received nivolumab $(3 \mathrm{mg} / \mathrm{kg}$, i.v. drip, day 1$)$ every 2 weeks and rh-endostatin $(210 \mathrm{mg}$, continuous i.v. infusion for $168 \mathrm{~h}$ ) every 4 weeks until disease progression or discontinuation. The primary endpoint was the objective response rate (ORR). The secondary endpoints included disease control rate (DCR), duration of response (DOR), clinical benefit response rate (CBR), progression-free survival (PFS), overall survival (OS) and safety.

Results: A total of 34 patients received a median of 4 cycles of therapy. In all, 14 patients achieved confirmed partial response (PR) with an ORR of 41.2\% [14/34; 95\% confidence interval (CI): 23.7-58.6\%], DCR of $64.7 \%$ (22/34; $95 \%$ CI: 47.8-81.6\%), CBR of 44.1\% (95\% CI: 26.5-61.7\%), and a DOR of 6.9 (95\% CI: 4.4-9.4) months. Median follow-up was 12.2 (range, 2.3-18.1) months. Median PFS (mPFS) was 6.8 (95\% CI: 1.1-12.1) months, median OS (mOS) was 17.1 (95\% CI: 6.6-27.6) months, and 12-month survival rate of $64.4 \%$ (95\% CI: 46.2-82.6\%). In all, 18 (18/34, 52.9\%) patients experienced at least one treatmentrelated adverse event (TRAE), and Grade 3 TRAEs occurred in 4 (4/34, 11.8\%) of them.

Conclusions: This study is first to assess nivolumab plus rh-endostatin in previously treated patients with advanced NSCLC. In view of its favorable efficacy and safety profile, this combination represents a promising treatment regimen in this patient population. 


\begin{abstract}
Keywords: Anti-angiogenesis; Endostar; immune checkpoint inhibitors (ICIs); nivolumab; non-small-cell lung cancer (NSCLC)
\end{abstract}

Submitted Nov 04, 2021. Accepted for publication Feb 16, 2022.

doi: $10.21037 /$ tlcr-22-49

View this article at: https://dx.doi.org/10.21037/tlcr-22-49

\section{Introduction}

Immune checkpoint inhibitors (ICIs) play an important role in the treatment of advanced non-small-cell lung cancer (NSCLC). Two phase III trials demonstrated that nivolumab vs. docetaxel improved overall survival (OS) and showed a favorable safety profile in patients with previously treated advanced NSCLC $(1,2)$, making nivolumab one of the current new standard second-line treatments in advanced NSCLC. Besides nivolumab, atezolizumab and pembrolizumab also have been approved as second-line treatments. A primary concern about ICI monotherapy as second-line therapy is the unsatisfactory tumor regression in clinical practice. To overcome this problem, combination therapy has been proposed and developed.

A series of preclinical studies suggest that ICIs and antiangiogenesis therapy may have synergistic antitumor effects (3-6). Anti-angiogenesis therapy not only normalizes the tumor vasculature but also optimizes the tumor's immune microenvironment (7). Similar to the promising findings from preclinical studies, many clinical trials have shown encouraging clinical benefits and a favorable safety profile for combining ICIs and antiangiogenic drugs in patients with advanced NSCLC (8-12). The IMPOWER150 study including atezolizumab, bevacizumab and chemotherapy has unlocked the door of combination therapy of immunotherapy and anti-angiogenic drug for first-line in advanced non-squamous NSCLC (10). In view of the hightoxicity from the IMPOWER150 trial, chemotherapy-free treatment combing with PD-1 inhibitor and anti-angiogenic has recently emerged as a novel treat pattern in first-line or later-line advanced NSCLC treatment $(8,9,11,12)$. But this choice is based on the evidence of small-size sample phase I/II clinical trials, the effectiveness of the combination therapy should be further confirmed by more well-designed double-blinded randomized controlled trials. In addition, the question of the optimal combination pattern and order of administration, the best candidates, and predictable bio-markers for combination of PD-1 inhibitor and antiangiogenic remain unresolved. Taken together, more favorable combinations remain to be investigated.

Recombinant human (rh)-endostatin (Endostar ${ }^{\circledR}$, Simcere Biopharmaceutical Co., Ltd., Jiangsu, China) can inhibit tumor endothelial cell proliferation, angiogenesis and tumor growth by down-regulating expression of vascular endothelial growth factors (VEGFs) and suppressing other important targets like matrix metalloproteinases (MMPs), hypoxia inducible factor 1 alpha (HIF-1 $\alpha$ ), basic fibroblast growth factor (bFGF) $(13,14)$. Therefore, rh-endostatin as a multiple-targets angiogenesis inhibitor has its own advantages compared to other anti-angiogenic agents, such as monoclonal antibodies (mAbs) and tyrosine kinase inhibitors (TKIs). A phase III trial suggested that rh-endostatin can improve the effect of the NP (vinorelbine and cisplatin) regimen with tolerable adverse effects in patients with untreated advanced NSCLC (15). According to previous results, rh-endostatin combined with chemotherapy received approval as a first-line treatment for advanced NSCLC in China. Moreover, preclinical studies have reported that rhendostatin normalizes the tumor vasculature and improves microenvironment to help carry drug and oxygen to tumor (16-18). These processes enhancing the antitumor activity of chemotherapy, as well as having synergistic antitumor effects with PD-1 blockade (4). Furthermore, compared to other anti-angiogenic drugs, rh-endostatin has been shown to have less severe toxic profiles and better compliance $(15,19,20)$. Especially, its safety and effectiveness in lung squamous cell carcinomas have been demonstrated $(15,20)$, where mAbs and TKIs lost approval due to safety concerns. Based on such data, we considered that the combination of rh-endostatin and nivolumab is a feasible treatment for later-line advanced NSCLC therapy, whatever its pathological type. So, we conducted this trial to assess the efficacy and safety of nivolumab plus rh-endostatin continuous intravenous infusion (CIV) as second-line or later treatment for patients with advanced NSCLC. We present the following article in accordance with the TREND reporting checklist (available at https://tlcr. amegroups.com/article/view/10.21037/tlcr-22-49/rc). 


\section{Methods}

\section{Study design}

This pilot study of nivolumab plus rh-endostatin in previously treated patients with NSCLC was conducted in The Fifth Affiliated Hospital of Sun Yat-sen University and the Affiliated Tumor Hospital of Guangxi Medical University, China from April 2019 to January 2021. The study protocol was approved by the institutional ethics committee of The Fifth Affiliated Hospital of Sun Yatsen University (No. K08-1), and informed consent was given by all enrolled patients. This trial was registered with the Chinese Clinical Trials Registry (Identifier: ChiCTR1900023664) and conducted in accordance with Good Clinical Practice guidelines and the Declaration of Helsinki (as revised in 2013). Regular evaluations of all patients were performed, which included physical examination, medical history, laboratory testing, electrocardiography (ECG), computed tomography (CT) of the thorax and abdomen, brain magnetic resonance imaging or CT, etc. The American Joint Committee on Cancer (AJCC, 8th edition) staging system was applied to all patients.

\section{Patient eligibility}

Eligible patients were $18-75$ years with histologically proven stage IV NSCLC and an Eastern Cooperative Oncology Group performance status (ECOG PS) score of $0-2$. Other eligibility criteria included adequate organ function, lesion evaluable by Response Evaluation Criteria in Solid Tumors (RECIST) criteria, and disease progression after at least one prior chemotherapy session alone or with anti-angiogenesis. Exclusion criteria included previously received ICI therapy, epithelial growth factor receptor $(E G F R)$ or anaplastic lymphoma kinase $(A L K)$ targetable mutations, active hemorrhage or at risk of hemorrhage, active known or suspected autoimmune disease, serious complications (uncontrolled infection, uncontrolled hypertension, respiratory failure, heart failure, hepatic failure or renal failure), allergic or possibly allergic to investigational drugs, perinatal females, and patients already participating in other clinical research.

\section{Treatment}

Nivolumab (Opdivo ${ }^{\circledR}$, Bristol Myers Squibb, USA) was intravenously administered at a dose of $3 \mathrm{mg} / \mathrm{kg}$ of body weight on day 1 every 2 weeks and rh-endostatin at a dose of $210 \mathrm{mg}$ CIV for 168 hours with an infusion pump every 4 weeks. Treatment continued until disease progression or treatment discontinuation for all patients. Other antitumor treatment was prohibited before disease progression. Therapy was interrupted when patients experienced Grade 4 toxicity and re-administered if toxicity recovered to $\leq$ Grade 2. Patients with intolerable adverse events (AEs) that delayed or caused discontinuation of one drug continued treatment with the other drug.

\section{Endpoints and measurements}

The primary endpoints were safety and the objective response rate (ORR), defined as the proportion of patients with complete response (CR) or partial response (PR). The secondary endpoints included the disease control rate (DCR), duration of response (DOR), clinical benefit response rate (CBR), progression-free survival (PFS), OS and safety. DCR was calculated as the proportion of patients with CR, PR and stable disease (SD). DOR was defined as the time between the date of first documented $\mathrm{CR}$ or PR and the date of first documented progression or death. CBR was defined as the proportion of patients with CR or PR or SD at week 24. PFS was defined as the time from registration to confirmed progressive disease (PD) or death. OS was defined as the time from patient enrollment to death. Investigators assessed treatment response according to RECIST 1.1 every 8 weeks during the first six cycles and every 2 months thereafter. Tumor response in the first assessment had to be confirmed by a subsequent radiographic assessment after $\geq 4$ weeks. Patients with $\mathrm{PD}$ in the first radiographic assessment were permitted to continue treatment, revealing that there were evaluated clinical benefits from continued treatment. After PD, they would be visited every 8 weeks for survival assessment. Safety was evaluated throughout the study. AEs including immune-related AEs (irAEs) were noted and graded per the U.S. National Cancer Institute's Common Terminology Criteria for Adverse Events (CTCAE V 4.03) and recorded from enrollment until disease progression.

\section{Statistical analysis}

All patients who received at least one dose of the investigational drugs were included in the efficacy and safety analyses. Safety was assessed in the safety analysis set (SAS). Efficacy was assessed in the full analysis set (FAS) and 


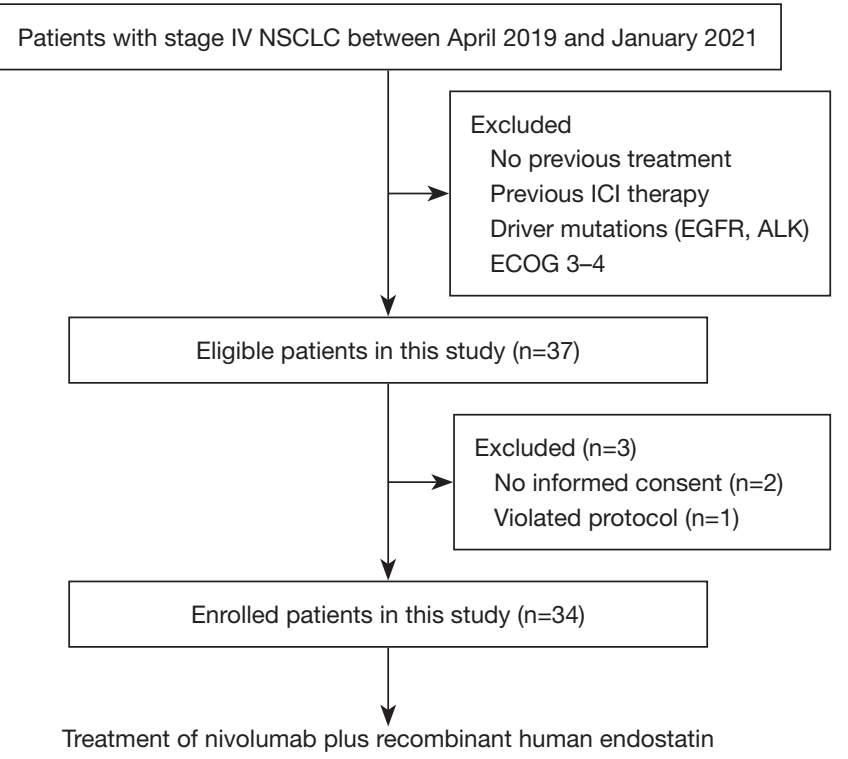

Figure 1 Study flow diagram. NSCLC, non-small-cell lung cancer; ICI, immune checkpoint inhibitor; EGFR, epithelial growth factor receptor; $A L K$, anaplastic lymphoma kinase; ECOG, Eastern Cooperative Oncology Group.

efficacy-evaluable set. All patients who received any dose of the investigational drugs were defined as the FAS. Subjects who received any dose of the investigational drugs and at least one radiographic assessment for efficacy were defined as the efficacy-evaluable set. Demographics, baseline characteristics, and tolerability were assessed by descriptive statistical analyses. Categorical data are described using numbers and percentages, and continuous variables are presented as mean \pm standard deviation or median (interquartile range). The $95 \%$ confidence interval (CI) for the ORR, DCR, DOR and CBR were calculated using the Clopper-Pearson method. PFS and OS were estimated using the Kaplan-Meier method. Subgroups were compared using Fisher's exact test for ORR and DCR, and two-sided log-rank test for PFS and OS. All data analysis was done with SPSS statistical software for Windows, version 22.0 (SPSS, Chicago, IL, USA).

\section{Results}

\section{Patients' characteristics}

Between April 23, 2019 and January 30, 2021, a total of 37 participants were screened for eligibility, but two patients withdrew their consent and one patient violated protocol before initial treatment. Finally, a total of 34 eligible patients with advanced NSCLC without targetable driver mutations received at least one dose of nivolumab and rhendostatin (Figure 1). All patients were previously treated by chemotherapy with or without anti-angiogenesis agents but were immunotherapy-naive. Of these patients, the median age was 60 (range, 37-72) years, 26.5\% were ECOG PS score 2 and $50.0 \%$ was current or former smokers. A total of $22(64.7 \%)$ had adenocarcinomas, 8 (23.5\%) had squamous cell carcinomas, and 4 was diagnosed as lymphoepitheliomalike carcinoma. Among the patients, 29.4\% had metastasis involving more than two organs, including 9 (26.5\%) with liver metastasis and $8(23.5 \%)$ with brain metastasis at baseline. In addition, $32.4 \%$ of patients received $>2$ prior systemic therapies and $47.1 \%$ of patients had received anti-angiogenesis treatment previously. The baseline characteristics of the participants are detailed in Table 1.

\section{Clinical efficacy}

Of the 34 patients, with a median follow-up of 12.2 (range, 2.3-18.1) months in the FAS, 14 (41.2\%) had confirmed PR, 8 (23.5\%) had SD, 12 (35.3\%) had PD (including three with unconfirmed PD based on clinical assessment without radiographic evaluation) (Table 2). In the efficacy-evaluable population $(\mathrm{n}=31)$, the confirmed ORR was $45.2 \%(14 / 31$; 95\% CI: $26.6-63.7 \%), 22$ patients had controlled disease, with a DCR of $71.0 \%$ (22/31; $95 \%$ CI: $54.0-87.9 \%)$.

Furthermore, 15 achieved CBR, with a CBR rate of $48.4 \%(15 / 31 ; 95 \%$ CI: 29.8-67.0\%) (Table 2). The detailed shrinkage of target lesions in the efficacyevaluable population is shown in Figure $2 \mathrm{~A}$. In addition, mean DOR was 6.9 (95\% CI: 4.4-9.4) months with treatment still ongoing in 8 patients at the time of data extraction (Figure 2B). In terms of ORR, the advantage of nivolumab plus rh-endostatin combination was observed in patients with ECOG PS score $0-1$ (56.0\% vs. $0.0 \%$; $\mathrm{P}=0.004)$. The DCR of nivolumab plus rh-endostatin treatment was also favorable in the patients with ECOG PS $0-1$ ( $80.0 \%$ vs. $22.2 \%$; $\mathrm{P}=0.004)$. However, patients in subgroups defined by sex, tumor histology, smoking status, number of prior systemic therapies, liver or brain metastasis, and prior anti-angiogenesis therapy had consistent ORR or DCR (Table 3).

Overall, the median PFS (mPFS) was 6.8 (95\% CI: 1.1-12.1) months (Figure 3A), median OS (mOS) was 17.1 (95\% CI: 6.6-27.6) months, and the 12 -month survival rate was $64.4 \%$ (95\% CI: 46.2-82.6\%) (Figure 3B). In 
Table 1 Baseline characteristics

\begin{tabular}{|c|c|}
\hline Characteristic & Total $(n=34)$ \\
\hline \multicolumn{2}{|l|}{ Age, years } \\
\hline Median [range] & 60 [37-72] \\
\hline \multicolumn{2}{|l|}{ Sex, n (\%) } \\
\hline Male & $23(67.6)$ \\
\hline Female & $11(32.4)$ \\
\hline \multicolumn{2}{|l|}{ ECOG PS, n (\%) } \\
\hline $0-1$ & $25(73.5)$ \\
\hline 2 & $9(26.5)$ \\
\hline \multicolumn{2}{|l|}{ Tumor histology, n (\%) } \\
\hline Adenocarcinoma & $22(64.7)$ \\
\hline Squamous & $8(23.5)$ \\
\hline Other & $4(11.8)$ \\
\hline \multicolumn{2}{|l|}{ Smoking status, n (\%) } \\
\hline Current or former smoker & $17(50.0)$ \\
\hline Never smoker & $17(50.0)$ \\
\hline \multicolumn{2}{|c|}{ No. of prior systemic therapies, $\mathrm{n}(\%)$} \\
\hline 1 & $8(23.5)$ \\
\hline 2 & $15(44.1)$ \\
\hline$>2$ & $11(32.4)$ \\
\hline \multicolumn{2}{|c|}{ No. of organs with metastasis, n (\%) } \\
\hline$\leq 2$ & $24(70.6)$ \\
\hline$>2$ & $10(29.4)$ \\
\hline \multicolumn{2}{|l|}{ Brain metastasis, $\mathrm{n}(\%)$} \\
\hline Yes & $8(23.5)$ \\
\hline No & $26(76.5)$ \\
\hline \multicolumn{2}{|l|}{ Liver metastasis, $\mathrm{n}(\%)$} \\
\hline Yes & $9(26.5)$ \\
\hline No & $25(73.5)$ \\
\hline \multicolumn{2}{|c|}{ Prior anti-angiogenesis therapy, $\mathrm{n}(\%)$} \\
\hline Yes & $16(47.1)$ \\
\hline No & $18(52.9)$ \\
\hline
\end{tabular}

ECOG PS, Eastern Cooperative Oncology Group performance status.

the subgroup analyses, there was no significant difference in the mPFS or mOS between the patients, regardless of sex, tumor histology, smoking status, number of prior treatments and liver metastasis (Table 3). Compared with ECOG PS 2 patients, ECOG PS $0-1$ patients had a longer mPFS (12.5 vs. 1.7 months; $\mathrm{P}<0.001)$ and mOS $(17.1 v s$. 3.4 months; $\mathrm{P}<0.001)$. Similarly, compared with the others, patients with $\leq 2$ metastatic organs achieved a longer mPFS (9.4 vs. 3.3 months; $\mathrm{P}=0.027$ ) and mOS (17.1 vs. 5.3 months; $\mathrm{P}=0.006)$. The patients without brain metastasis had better mPFS (7.5 vs. 1.8 months; $\mathrm{P}=0.037)$ and $\operatorname{mOS}(17.1$ vs. 5.3 months; $\mathrm{P}=0.029)$ than the patients with brain metastasis. Nevertheless, the patients who never received anti-angiogenesis therapy had better mPFS (12.5 vs. 2.8 months; $\mathrm{P}=0.021$ ) yet similar mOS $[17.1$ months $v s$. not reached $(\mathrm{NR}) ; \mathrm{P}=0.112]$.

\section{AEs}

The median duration of nivolumab plus rh-endostatin therapy was 4.0 (range, 1-14) cycles. Among all enrolled patients, 18 (52.9\%) experienced at least one treatmentrelated AE (TRAE), and Grade 3 TRAEs occurred in 4 (11.8\%) of them (Table 4). The most common TRAEs were hypothyroidism (7, 20.6\%), arrhythmia (5, 14.7\%), immune-related pneumonitis $(3,8.8 \%)$, proteinuria $(3$, $8.8 \%)$, abnormal hepatic function $(2,5.9 \%)$, and increased blood creatinine (2, 5.9\%). The Grade 3 TRAEs comprised immune-related pneumonitis $(2,5.9 \%)$, atrial fibrillation $(1,2.9 \%)$, and catheter-related infection $(1,2.9 \%)$. Three patients $(3,8.8 \%)$ discontinued treatment because of immune-related pneumonitis or catheter-related infection, and one patient delayed treatment for atrial fibrillation. No unexpected AEs were observed.

\section{Discussion}

In this phase II study, we evaluated the combination of nivolumab plus rh-endostatin as second-line or later treatment in patients with advanced NSCLC without sensitive mutations. This exploratory trial demonstrated that this combined regimen had promising efficacy and favorable tolerability.

PD-1/PD-L1 blockade immunotherapy has recently been defined as the new standard second-line treatment for advanced NSCLC according to the results from a series of important randomized controlled clinical trials (1,2,21-23). Currently, pembrolizumab, nivolumab, and atezolizumab have been approved for second-line treatment by the U.S. FDA based on longer OS benefit in advanced NSCLC patients. Of these, nivolumab is the only approved 
Table 2 Best overall tumor response of investigator-evaluation

\begin{tabular}{lcc}
\hline Outcome & All patients $(n=34)$ & Efficacy-evaluable patients $(n=31)$ \\
\hline Median follow-up (range), months & $12.2(2.3-18.1)$ & - \\
Best overall response, $n$ (\%) & & $0(0.0)$ \\
CR & $0(0.0)$ & $14(45.2)$ \\
PR & $14(41.2)$ & $8(25.8)$ \\
SD & $8(23.5)$ & $9(29.0)$ \\
PD & $12(35.3)$ & $45.2(26.6-63.7)$ \\
ORR, \% (95\% Cl) & $41.2(23.7-58.6)$ & $71.0(54.0-87.9)$ \\
DCR, \% (95\% Cl) & $64.7(47.8-81.6)$ & $48.4(29.8-67.0)$ \\
CBR, \% (95\% Cl) & $44.1(26.5-61.7)$ & $6.9(4.4-9.4)$ \\
DOR, months (95\% Cl) & $6.9(4.4-9.4)$ & \\
\hline
\end{tabular}

$\mathrm{CR}$, complete response; PR, partial response; SD, stable disease; PD, progressive disease; ORR, objective response rate; Cl, confidence interval; DCR, disease control rate; CBR, clinical benefit response rate; DOR, duration of response.

immunotherapy drug for this patient population in China. Results from several preclinical trials suggest that antiangiogenesis could enhance the antitumor effects of ICIs by normalizing the tumor vasculature and improving tumor immune microenvironment (3-6). Presently, numerous clinical trials have focused on the combination of antiangiogenic agents and ICIs to investigate the efficacy in gastric cancer, gastroesophageal junction adenocarcinoma, hepatocellular carcinoma, and renal cell cancer, as well as NSCLC (24). In particular, the synergy of PD-1 blockade and rh-endostatin has been verified in a lung cancer mouse model $(4,17,25)$. Clearly, the exploratory study of nivolumab plus rh-endostatin in advanced NSCLC is based on the large amount of preclinical evidence.

In the current clinical study, despite the baseline clinical features of poor ECOG PS, multiple organ metastasis, and heavily pretreated enrolled patients, the ORR, DCR, and survival of the patients given nivolumab plus rh-endostatin were satisfactory. Notably, the addition of rh-endostatin to nivolumab increased the ORR to $41.2 \%$ and the DCR to $64.7 \%$, better than the ORR (16.6-20.0\%) and the DCR (44.0-52.4\%) of nivolumab alone in the CheckMate 017, CheckMate 057, and CheckMate 078 trials $(1,2,21)$. Although cross-study comparison can be controversial, the other efficacy endpoints, including mPFS and mOS, of the combination therapy in this study also seem to be more favorable than nivolumab monotherapy in the above three trials (mPFS: 6.8 vs. 2.3-3.5 months and mOS: 17.1 vs. 9.2-12.2 months) $(1,2,21)$. Moreover, the 12 -month survival rate with nivolumab plus rh-endostatin was also higher than for nivolumab alone (64.4\% vs. $42-50 \%)(1,2,21)$. Additionally, compared to other ICI monotherapy trials, including the OAK trial (22) and KEYNOTE-010 (23), a higher ORR, DCR and better survival were also noted in this trial. Certainly, the advantage of nivolumab and rh-endostatin combination therapy compared to nivolumab monotherapy as second-line treatment of advanced NSCLC warrants further confirmation by a randomized controlled phase III clinical study.

Recently, the clinical benefits of combining antiangiogenesis with ICIs in first-line or later treatment have emerged in several clinical trials of lung cancer (8-12). A phase Ib trial reported that sintilimab plus anlotinib exhibited a highly superior ORR (72.7\%) and DCR (100\%) as first-line treatment in advanced NSCLC (8), making chemotherapy-free management possible in this patient population. In term of previously treated NSCLC patients with ECOG PS of $0-1$, the combination of ramucirumab and pembrolizumab in the JVDF study, which included 27 patients, exhibited an ORR of $30 \%$, DCR of $85 \%$ and a 12 -month OS rate of $68 \%$ (9). Consistent with those results, similarly high ORR, DCR and survival rate were also observed in two phase Ib/II trials with lowdose apatinib plus camrelizumab (11) or lenvatinib plus pembrolizumab (12). The efficacy results in the above trials were numerically similar to those of the overall population in our study. But in this trial, the proportions of patients with ECOG PS of 2 (26.5\%), liver metastasis (26.5\%), brain metastasis $(23.5 \%)$, and prior anti-angiogenesis 

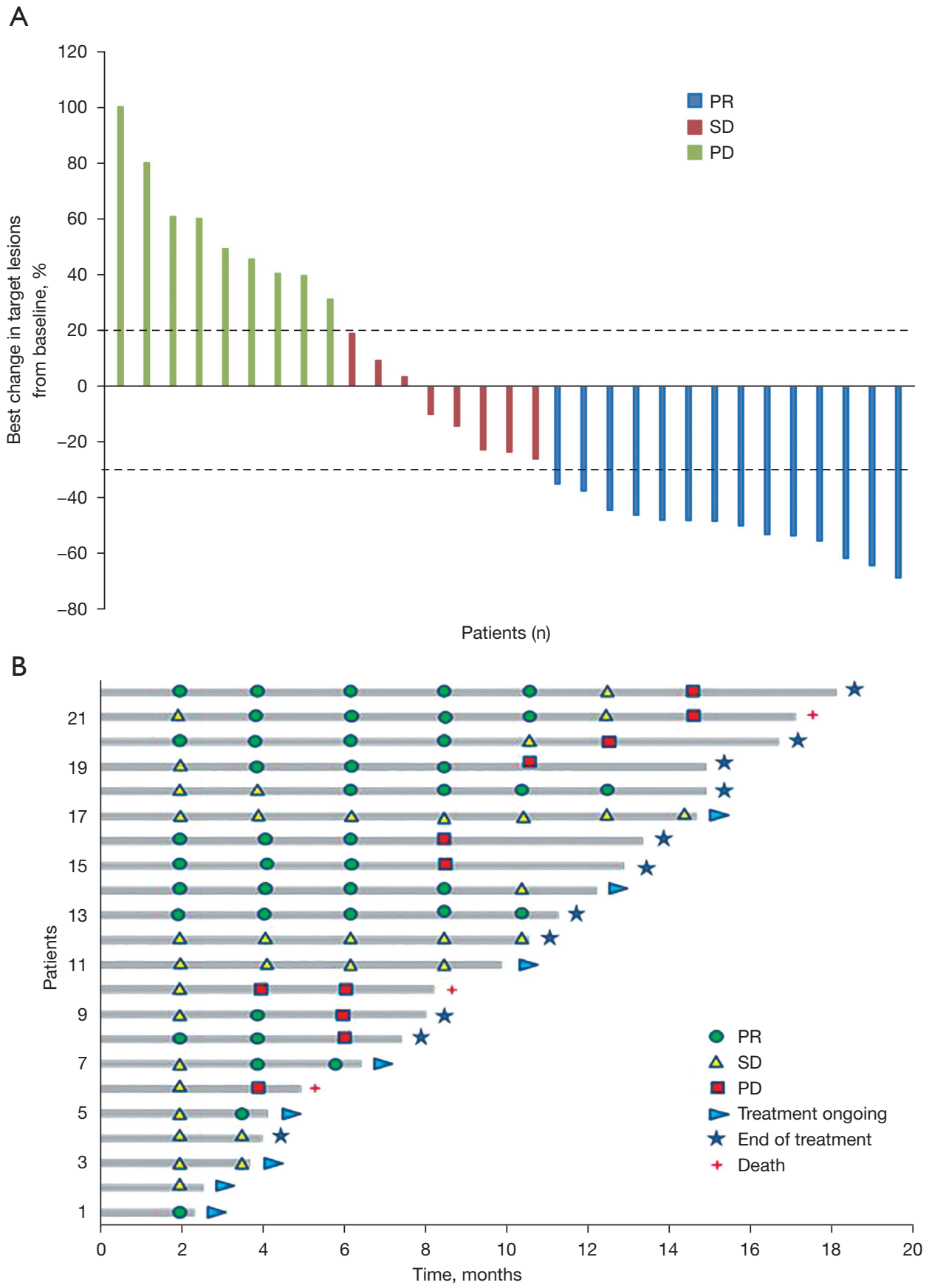

Figure 2 Investigator-assessed efficacy of nivolumab combined with rh-endostatin. (A) Best responses in efficacy-evaluable patients (n=31). (B) Duration of treatment in patients with objective response $(n=22)$. PR, partial response; SD, stable disease; PD, progressive disease.

therapy $(47.1 \%)$ were higher than in the other trials. In our subgroup analysis of ECOG PS 0-1, which included 25 patients, the ORR was $56.0 \%(14 / 25)$, the DCR was $80.0 \%$ (20/25) (Table 3). The efficacy endpoints in ECOG PS 0-1 patients were better than for ICIs plus anti-angiogenesis agents in previous studies $(9,11,12)$. ECOG PS 2 and liver metastasis showed a poor response to immunotherapy, which was similar to other studies $(10,23,26)$. It is noteworthy that prior anti-angiogenesis therapy may have affected the tumor response and mPFS of nivolumab plus 
Table 3 ORR, PFS and OS in all subgroup analyses in FAS

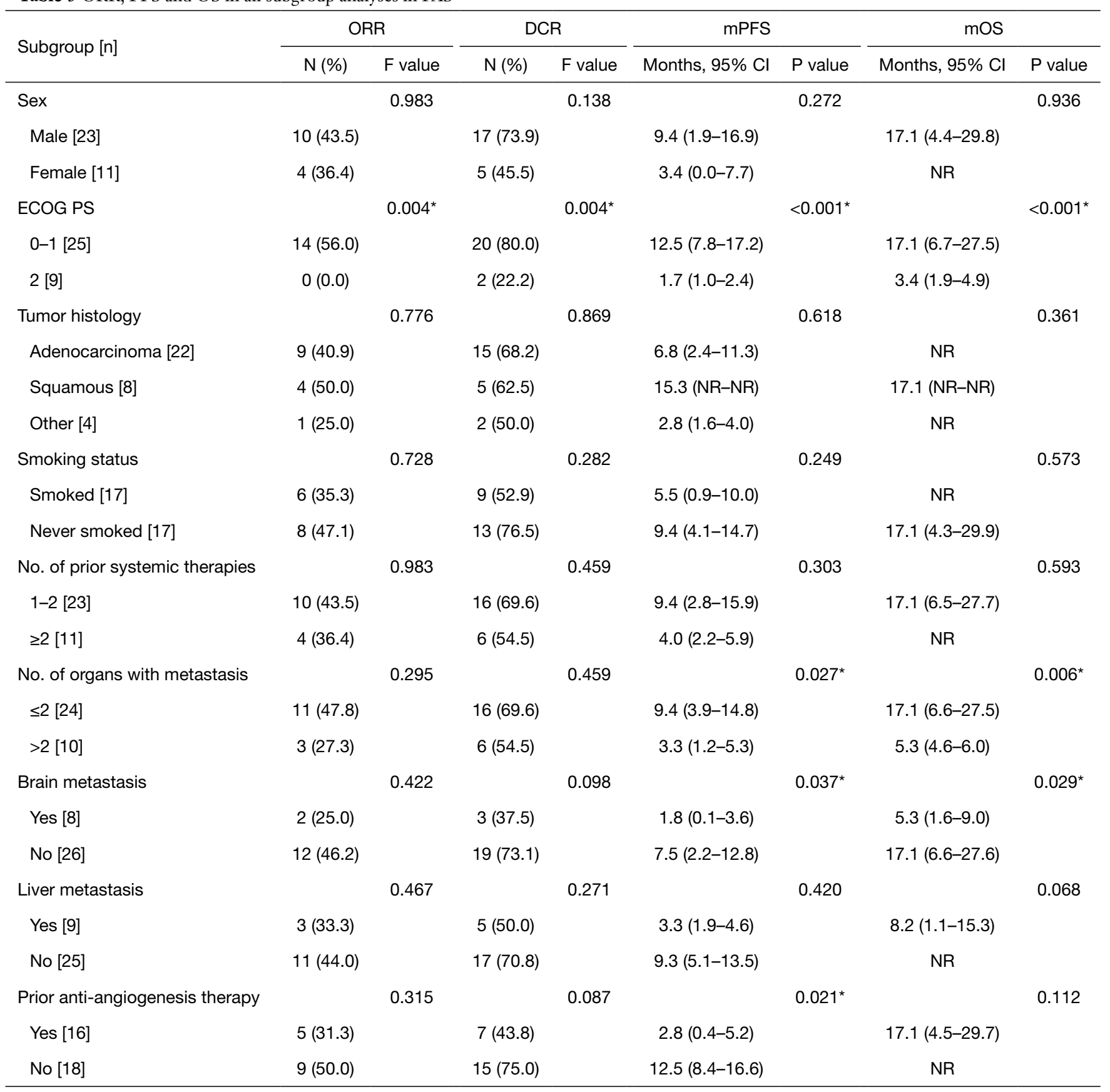

*, represent $\mathrm{P}$ values with significant difference. ORR, objective response rate; PFS, progression-free survival; OS, overall survival; FAS, full analysis set; DCR, disease control rate; mPFS, median PFS; mOS, median OS; ECOG PS, Eastern Cooperative Oncology Group performance status; NR, not reached.

rh-endostatin, but not the mOS.

Undoubtedly, anti-angiogenic agents increased the risk of hypertension, cardiovascular events, proteinuria, and arterial thromboembolism $(27,28)$. Meanwhile, hypothyroidism, pneumonitis, arthralgia and skin irAEs are more common with PD-1 mAbs $(1,2,21)$. Theoretically, the degrees and frequencies of the two above-mentioned toxicities may be increased accordingly. According to previous studies, 

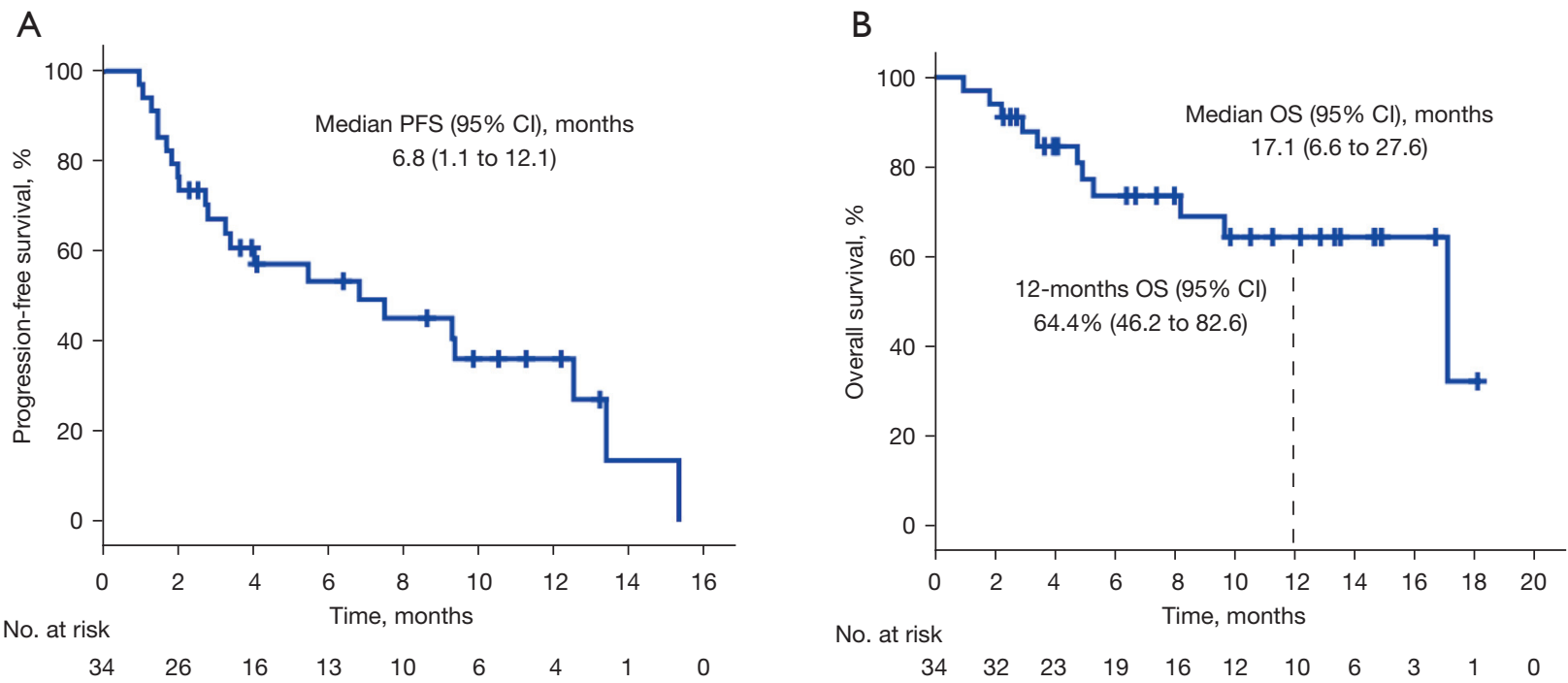

Figure 3 Kaplan-Meier graph for survival in all patients. (A) PFS in all patients. (B) OS in all patients. PFS, progression-free survival; OS, overall survival; CI, confidence interval.

Table 4 TRAEs in SAS

\begin{tabular}{|c|c|c|c|}
\hline AEs & \multicolumn{3}{|c|}{ All patients, $\mathrm{n}(\%)$} \\
\hline Hypothyroidism & $7(20.6)$ & $7(20.6)$ & $0(0.0)$ \\
\hline Arrhythmia & $5(14.7)$ & $4(11.8)$ & $1(2.9)$ \\
\hline Atrial premature beats & $2(5.9)$ & $2(5.9)$ & $0(0.0)$ \\
\hline Atrial fibrillation & $1(2.9)$ & $0(0.0)$ & $1(2.9)$ \\
\hline Immune-related pneumonitis & $3(8.8)$ & $1(2.9)$ & $2(5.9)$ \\
\hline Abnormal hepatic function & $2(5.9)$ & $2(5.9)$ & $0(0.0)$ \\
\hline Blood creatinine increased & $2(5.9)$ & $2(5.9)$ & $0(0.0)$ \\
\hline Hyperglycemia & $2(5.9)$ & $2(5.9)$ & $0(0.0)$ \\
\hline Creatine kinase & $2(5.9)$ & $2(5.9)$ & $0(0.0)$ \\
\hline Hypertension & $2(5.9)$ & $2(5.9)$ & $0(0.0)$ \\
\hline Rash & $1(2.9)$ & $1(2.9)$ & $0(0.0)$ \\
\hline Left ventricular ejection fraction decreased & $1(2.9)$ & $1(2.9)$ & $0(0.0)$ \\
\hline Catheter-related infection & $1(2.9)$ & $0(0.0)$ & $1(2.9)$ \\
\hline Any $A E$ & 18 (52.9) & $14(41.1)$ & $4(11.8)$ \\
\hline
\end{tabular}

TRAE, treatment-related adverse event; SAS, safety analysis set; AE, adverse event. 
both types of therapy will result in an increased risk of toxicities simultaneously or respectively $(8,9,11)$. In current study, there were no new or unexpected safety signal and tolerability issues with the combination of nivolumab and rhendostatin. Neither Grade 4 TRAEs nor treatment-related deaths occurred. Grade 3-5 TRAEs occurred in $11.4 \%$ of patients, but their incidence has been approximately $60 \%$ in other studies of ICIs plus anti-angiogenesis $(8,9,11,12)$. The incidences of any grade and Grade 3 immune-related pneumonitis were respectively $8.8 \%(3 / 34)$ and $5.9 \%(2 / 34)$, which was higher than those reported for nivolumab alone $(1,2,21,29)$. Grade $1-2$ hypothyroidism occurred in $20.6 \%$ of the patients in this study, which was comparable to previous studies $(8,9,11,12)$. Another predominant TRAE was arrhythmia (14.7\%): Grade $1-2$ was $11.8 \%$ and Grade 3 was $2.9 \%$. The incidence of arrhythmia was similar to that for rh-endostatin combined with chemotherapy $(15,19,20,30,31)$. CIV of rh-endostatin is a novel method of administration that had similar efficacy and safety but was more convenient than the traditional intermittent intravenous infusion method (19,32); however, it may increase the risk of catheter-related infection: $8.8 \%(3 / 34)$ of patients discontinued treatment because of immune-related pneumonitis or catheter-related infection.

The limitations in the current study cannot be neglected. First, the study was an open-label, single-arm study with limited sample size, which might result in bias without a nivolumab monotherapy control arm. Therefore, a randomized, nivolumab-controlled clinical trial with bigger sample size will be carried out for further verification of our findings in the future. Second, the PD-1 expression assay, $V E G F$, tumor mutational burden (TMB) or other biomarkers were not validated for exploratory analysis of efficacy. Third, the results cannot be generalized because of the heterogeneity of the study population.

\section{Conclusions}

In short, this study revealed that nivolumab combined with rh-endostatin as second-line or later treatment showed promising efficacy and favorable tolerability in pretreated advanced NSCLC patients.

\section{Acknowledgments}

The authors appreciate the academic support from the AME Lung Cancer Collaborative Group. We thank all patients and their relatives for their participation, as well as all investigators and their staffs for their collaboration in this study. We presented parts of this study as an abstract at the 2021 ASCO Annual Meeting.

Funding: This study was supported by (I) the Investigator Initiated Trial Program of The Fifth Affiliated Hospital of Sun Yat-sen University (grant No. YNZZ 2020-05), (II) the Fundamental Research Funds for the Central Universities, Sun Yat-sen University (grant No. 2021qntd35), (III) the Science and Technology Project Grant of Zhuhai (ZH22036201210067PWC), (IV) the Scientific Research Project funded by Traditional Chinese Medicine Bureau of Guangdong Province (202106080515386340), (V) Investigator Initiated Trials of ChiCTR2000035079 (YNZZ 2021-03).

\section{Footnote}

Reporting Checklist: The authors have completed the TREND reporting checklist. Available at https://tlcr. amegroups.com/article/view/10.21037/tlcr-22-49/rc

Data Sharing Statement: Available at https://tlcr.amegroups. com/article/view/10.21037/tlcr-22-49/dss

Conflicts of Interest: All authors have completed the ICMJE uniform disclosure form (available at https://tlcr.amegroups. com/article/view/10.21037/tlcr-22-49/coif). The authors have no conflicts of interest to declare.

Ethical Statement: The authors are accountable for all aspects of the work in ensuring that questions related to the accuracy or integrity of any part of the work are appropriately investigated and resolved. The study protocol was approved by the institutional ethics committee of The Fifth Affiliated Hospital of Sun Yat-sen University (No. K08-1), and informed consent was given by all enrolled patients. This trial was registered with the Chinese Clinical Trials Registry (Identifier: ChiCTR1900023664) and conducted in accordance with Good Clinical Practice guidelines and the Declaration of Helsinki (as revised in 2013).

Open Access Statement: This is an Open Access article distributed in accordance with the Creative Commons Attribution-NonCommercial-NoDerivs 4.0 International License (CC BY-NC-ND 4.0), which permits the noncommercial replication and distribution of the article with the strict proviso that no changes or edits are made and the original work is properly cited (including links to both the 
formal publication through the relevant DOI and the license). See: https://creativecommons.org/licenses/by-nc-nd/4.0/.

\section{References}

1. Brahmer J, Reckamp KL, Baas P, et al. Nivolumab versus Docetaxel in Advanced Squamous-Cell Non-Small-Cell Lung Cancer. N Engl J Med 2015;373:123-35.

2. Borghaei H, Paz-Ares L, Horn L, et al. Nivolumab versus Docetaxel in Advanced Nonsquamous Non-Small-Cell Lung Cancer. N Engl J Med 2015;373:1627-39.

3. Yang Y, Li L, Jiang Z, et al. Anlotinib optimizes antitumor innate immunity to potentiate the therapeutic effect of PD-1 blockade in lung cancer. Cancer Immunol Immunother 2020;69:2523-32.

4. Wu J, Zhao X, Sun Q, et al. Synergic effect of PD-1 blockade and endostar on the PI3K/AKT/mTOR-mediated autophagy and angiogenesis in Lewis lung carcinoma mouse model. Biomed Pharmacother 2020;125:109746.

5. Zhao S, Ren S, Jiang T, et al. Low-Dose Apatinib Optimizes Tumor Microenvironment and Potentiates Antitumor Effect of PD-1/PD-L1 Blockade in Lung Cancer. Cancer Immunol Res 2019;7:630-43.

6. Di Tacchio M, Macas J, Weissenberger J, et al. Tumor Vessel Normalization, Immunostimulatory Reprogramming, and Improved Survival in Glioblastoma with Combined Inhibition of PD-1, Angiopoietin-2, and VEGF. Cancer Immunol Res 2019;7:1910-27.

7. Yi M, Jiao D, Qin S, et al. Synergistic effect of immune checkpoint blockade and anti-angiogenesis in cancer treatment. Mol Cancer 2019;18:60.

8. Chu T, Zhong R, Zhong H, et al. Phase 1b Study of Sintilimab Plus Anlotinib as First-line Therapy in Patients With Advanced NSCLC. J Thorac Oncol 2021;16:643-52.

9. Herbst RS, Arkenau HT, Santana-Davila R, et al. Ramucirumab plus pembrolizumab in patients with previously treated advanced non-small-cell lung cancer, gastro-oesophageal cancer, or urothelial carcinomas (JVDF): a multicohort, non-randomised, open-label, phase 1a/b trial. Lancet Oncol 2019;20:1109-23.

10. Socinski MA, Jotte RM, Cappuzzo F, et al. Atezolizumab for First-Line Treatment of Metastatic Nonsquamous NSCLC. N Engl J Med 2018;378:2288-301.

11. Zhou C, Wang Y, Zhao J, et al. Efficacy and Biomarker Analysis of Camrelizumab in Combination with Apatinib in Patients with Advanced Nonsquamous NSCLC Previously Treated with Chemotherapy. Clin Cancer Res 2021;27:1296-304.
12. Taylor MH, Lee CH, Makker V, et al. Phase IB/II Trial of Lenvatinib Plus Pembrolizumab in Patients With Advanced Renal Cell Carcinoma, Endometrial Cancer, and Other Selected Advanced Solid Tumors. J Clin Oncol 2020;38:1154-63.

13. Ling Y, Yang Y, Lu N, et al. Endostar, a novel recombinant human endostatin, exerts antiangiogenic effect via blocking VEGF-induced tyrosine phosphorylation of KDR/Flk1 of endothelial cells. Biochem Biophys Res Commun 2007;361:79-84.

14. Li K, Shi M, Qin S. Current Status and Study Progress of Recombinant Human Endostatin in Cancer Treatment. Oncol Ther 2018;6:21-43.

15. Sun Y, Wang JW, Liu YY, et al. Long-term results of a randomized, double-blind, and placebo-controlled phase III trial: Endostar (rh-endostatin) versus placebo in combination with vinorelbine and cisplatin in advanced non-small cell lung cancer. Thorac Cancer 2013;4:440-8.

16. Huang G, Chen L. Recombinant human endostatin improves anti-tumor efficacy of paclitaxel by normalizing tumor vasculature in Lewis lung carcinoma. J Cancer Res Clin Oncol 2010;136:1201-11.

17. Liu $X$, Nie $W, X i e ~ Q$, et al. Endostatin reverses immunosuppression of the tumor microenvironment in lung carcinoma. Oncol Lett 2018;15:1874-80.

18. Jiang XD, Dai P, Wu J, et al. Effect of recombinant human endostatin on radiosensitivity in patients with non-small-cell lung cancer. Int J Radiat Oncol Biol Phys 2012;83:1272-7.

19. Wang B, Xu L, Li Q, et al. Endostar continuous versus intermittent intravenous infusion combined with chemotherapy for advanced NSCLC: a systematic review and meta-analysis including non-randomized studies. BMC Cancer 2020;20:1021.

20. Han B, Xiu Q, Wang H, et al. A multicenter, randomized, double-blind, placebo-controlled study to evaluate the efficacy of paclitaxel-carboplatin alone or with endostar for advanced non-small cell lung cancer. J Thorac Oncol 2011;6:1104-9.

21. Wu YL, Lu S, Cheng Y, et al. Nivolumab Versus Docetaxel in a Predominantly Chinese Patient Population With Previously Treated Advanced NSCLC: CheckMate 078 Randomized Phase III Clinical Trial. J Thorac Oncol 2019;14:867-75.

22. Rittmeyer A, Barlesi F, Waterkamp D, et al. Atezolizumab versus docetaxel in patients with previously treated non-small-cell lung cancer (OAK): a phase 3, openlabel, multicentre randomised controlled trial. Lancet 2017;389:255-65. 
23. Herbst RS, Baas P, Kim DW, et al. Pembrolizumab versus docetaxel for previously treated, PD-L1-positive, advanced non-small-cell lung cancer (KEYNOTE-010): a randomised controlled trial. Lancet 2016;387:1540-50.

24. Song Y, Fu Y, Xie Q, et al. Anti-angiogenic Agents in Combination With Immune Checkpoint Inhibitors: A Promising Strategy for Cancer Treatment. Front Immunol 2020;11:1956.

25. Xu Q, Gu J, Lv Y, et al. Angiogenesis for tumor vascular normalization of Endostar on hepatoma 22 tumor-bearing mice is involved in the immune response. Oncol Lett 2018;15:3437-46.

26. Lobefaro R, Viscardi G, Di Liello R, et al. Immunotherapy in advanced Non-Small Cell Lung Cancer patients with poor performance status: The role of clinical-pathological variables and inflammatory biomarkers. Lung Cancer 2021;152:165-73.

27. Faruque LI, Lin M, Battistella M, et al. Systematic review of the risk of adverse outcomes associated with vascular endothelial growth factor inhibitors for the treatment of cancer. PLoS One 2014;9:e101145.

28. Kulke MH, Bergsland EK, Ryan DP, et al. Phase II study of recombinant human endostatin in patients with advanced neuroendocrine tumors. J Clin Oncol 2006;24:3555-61.

29. Khunger M, Rakshit S, Pasupuleti V, et al. Incidence of Pneumonitis With Use of Programmed Death 1 and Programmed Death-Ligand 1 Inhibitors in Non-Small Cell Lung Cancer: A Systematic Review and MetaAnalysis of Trials. Chest 2017;152:271-81.

30. Yang H, Sui Y, Guo X, et al. Endostar continuous intravenous infusion combined with $\mathrm{S}-1$ and oxaliplatin chemotherapy could be effective in treating liver metastasis from gastric cancer. J Cancer Res Ther 2018;14:S1148-51.

31. Li X, Gu G, Soliman F, et al. The Evaluation of Durative Transfusion of Endostar Combined with Chemotherapy in Patients with Advanced Non-Small Cell Lung Cancer. Chemotherapy 2018;63:214-9.

32. Cheng Y, Nie L, Liu Y, et al. Comparison of Endostar continuous versus intermittent intravenous infusion in combination with first-line chemotherapy in patients with advanced non-small cell lung cancer. Thorac Cancer 2019;10:1576-80.

(English Language Editor: Kerry. Brown)
Cite this article as: Lv W, Pei X, Zhao W, Cong Y, Wei Y, Li T, Zhang H, Lin Z, Saito Y, Kim JJ, Liang Z, Zhong B, Wang Z. Safety and efficacy of nivolumab plus recombinant human endostatin in previously treated advanced non-small-cell lung cancer. Transl Lung Cancer Res 2022;11(2):201-212. doi: $10.21037 /$ tlcr-22-49 